\title{
Magnetoconductance Oscillations in Ballistic Semiconductor-Superconductor Junctions
}

\author{
Yasuhiro Asano* \\ Department of Applied Physics, Hokkaido University, Sapporo 060-8628, Japan.
}

(June 27, 2018)

The mechanism of the magnetoconductance oscillations in junctions of a ballistic semiconductor and a superconductor is discussed. The oscillations appear when both the normal and the Andreev reflection occur at the interface. The interplay between the classical cyclotron motion of a quasiparticle and the phase shift caused by the magnetic field is the origin of the conductance oscillations.

PACS: 74.50.+r, 74.80.-g, 72.10.-d, 72.20.-i

Conductance oscillations as a function of the applied magnetic field in a small ring are a one of fundamental consequence of the phase-coherent transportl. The width of the ring must be narrow so that the number of the propagation path of an electron wave can be limited. If many propagation paths are allowed in the ring, the magnetoconductance oscillations (MCO) are washed out. In order to observe the MCO clearly, at least, we have to either confine the electron wave as in the experiment or the magnetic flux as in the original ideal. An electron wave, however, can be confined within a classical trajectory of the cyclotron motion by its charge degree of freedom under the relatively stron magnetic fields, which is called the magnetic focusing 3 . In the ballistic transport regime, we show a possibility to observe the MCO in simply-connected system.

In this paper, we discuss the conductance in small semiconductor-superconductor (Sm-S) junctions, where a high mobility two-dimensional electron gas (2DEG) is used as a semiconductor. Recently S-Sm-S junctions can be realized in strong magnetic fields $\mathrm{E}$. It has numerically shown that the sinusoidal MCO appears in rela tively weak magnetic fields when the Andreev reflection 6 is not perfectl. To date, however, the mechanism of the sinusoidal MCO has remained unclear. In this paper, we reveal the nature of the novel $\mathrm{MCO}$ within a simple analysis. We conclude that the interplay between the cyclotron motion of a quasiparticle (QP) and the phase shift caused by the magnetic field is responsible for the $\mathrm{MCO}$ in simply-connected Sm-S junctions. The MCO is one of the interference effect of the Andreev reflected QP, which have been focused recently 6 .11.

Let us consider a two-dimensional wire where electrons are confined in the $y$ direction in the range of $-W / 2<$ $y<W / 2$. The wire consists of 2DEG $(x<0)$ and S $(x>$ $0)$. The Sm-S junctions are described by the Bogoliubovde Gennes equation 12

$$
\left(\begin{array}{cc}
H_{0} & \Delta(x, y) \\
\Delta(x, y)^{*} & -H_{0}^{*}
\end{array}\right)\left(\begin{array}{l}
u \\
v
\end{array}\right)=E\left(\begin{array}{l}
u \\
v
\end{array}\right)
$$

where $u(x, y)$ and $v(x, y)$ are the wavefunctions of a quasiparticle. The Hamiltonian is given by $H_{0}=$ $-\hbar^{2}\{\nabla-i e \mathbf{A}(x, y) / \hbar c\}^{2} / 2 m^{*}+U(x, y)-\mu$, where the mass of an electron $m^{*}$ is $m_{N}$ for $x \leq 0$ and $m_{S}$ for $x>0$, respectively. The chemical potential of the junction is denoted by $\mu$. In what follows we set the chemical potential as an origin of the energy, i.e., $\mu=0$. The Fermi energy in 2DEG and $\mathrm{S}$ are $\mu_{N}$ and $\mu_{S}$, respectively, which correspond to the energy difference between the band edge and the chemical potential. The scalar potential $U(x, y)$ involves the hard wall confinement potential in the $y$ direction and the potential barrier at the $\mathrm{Sm}-\mathrm{S}$ interface which is described by $H \delta(x)$. The potential barrier height should be determined consistently by the the electronic structure on either sides of the junction ${ }^{13}$. In this paper, however, we treat $H$ as one of the independent parameters of Sm-S junctions. We assume that pair potential $\Delta(x, y)$ is $\Delta_{0}$ in $\mathrm{S}$ and zero in $2 \mathrm{DEG}$, respectively. This model is justified when the superconducting segment is covered with materials with high magnetic permeahil ity, because the magnetic field is not applied onto $\mathrm{S} 44$. Since $\mathrm{S}$ is magnetically shielded, the vector potential is $\mathbf{A}=(0,0)$ for $x \geq 0$ and $\mathbf{A}=(0, B x)$ for $x<0$. In what follows, we measure the energy and the length in units of $\mu_{N}$ and $1 / k_{F} \equiv \sqrt{2 m \mu_{N}} / \hbar$, respectively.

The wavefunction in 2DEG and that in S can be obtained separately, and are related with each other by using the continuity condition at $x=0$. The detail of the numerical simulations is given elsewhere 15 . Here we show the expression of the zero-bias conductance at zero temperature 1 , $G=\left(2 e^{2} / h\right) \sum_{l, n}{ }^{\prime}\left(\delta_{l, n}-R_{l, n}^{e e}+R_{l, n}^{h e}\right)$, where $l$ and $n$ label the propagation channels in 2DEG under the magnetic field, $R_{l, n}^{e e}$ and $R_{l, n}^{h e}$ are the reflection probability into the electron and hole branches with $E \rightarrow 0$, respectively. The summation $\sum_{l}{ }^{\prime}=N_{c}$ runs over the all propagating channels. In the limit of $E \rightarrow 0$, there is no propagating channel in $\mathrm{S}$. The current conservation low implies $\sum_{l}^{\prime}\left(R_{l, n}^{e e}+R_{l, n}^{h e}\right)=1$.

In Fig. 1, we show the numerical results of the conductance in units of $2 e^{2} / h$ as a function of $\beta \equiv \mu_{N} / \hbar \omega_{c}$, where $\omega_{c}=e B / \mathrm{cm}_{N}$ and $\Delta_{0} / \mu_{N}=0.02$, respectively. Since the pair potential in $\mathrm{S}$ is typically $1 \mathrm{meV}$ and the Fermi energy in 2DEG is about $100 \mathrm{meV}$, we fix $\Delta_{0} / \mu_{N}$ at 0.02 throughout this paper. We also fix the width of the wire $W k_{F}$ at 40 . The numerical results of the conductance presented here are essentially the same with those 
in Ref. 7. There are three parameters which characterize the Sm-S junctions and the reflection probability at the interface:(i) the difference of the Fermi energy, $\mu_{S} / \mu_{N}$, (ii) the difference of the effective mass, $m_{S} / m_{N}$, and (iii) the potential barrier, $V_{b h} \equiv 2 m_{N} H / \hbar^{2} k_{F}$. In the the solid line of Fig. 1 (a), we show the conductance for $\mu_{S} / \mu_{N}=1, m_{S} / m_{N}=1$ and $V_{b h}=0$. The results show the conductance step and the conductance decreases with increasing the magnetic field. In this case, we have confirmed that the normal reflection does not occur at the interface from numerical data, i.e., $R_{l, n}^{e e} \simeq 0$. This can be also understood by the S-matrix in one-dimensional Sm-S interface

$$
\begin{aligned}
& r_{e e}=\sqrt{\left(1-|\xi|^{2}\right)^{2}+(2 \operatorname{Im} \xi)^{2}} /\left(1+|\xi|^{2}\right) \mathrm{e}^{i \theta_{n}}=r_{h h}^{*}, \\
& r_{h e}=2 \operatorname{Re} \xi /\left(1+|\xi|^{2}\right) \mathrm{e}^{-i \pi / 2}=r_{e h},
\end{aligned}
$$

where $\xi \simeq \sqrt{\left(m_{N} / m_{S}\right)\left(\mu_{S} / \mu_{N}\right)}+i V_{b h}$ and $\tan \theta_{n} \simeq$ $2 V_{b h} /\left[\left(m_{N} / m_{S}\right) \cdot\left(\mu_{S} / \mu_{N}\right)-1+V_{b h}^{2}\right]$. Here we solve Eq. (11) in one-dimension and calculate the reflection coefficients in the limit of $\Delta_{0} \ll \mu_{N}$ and $E=0$. In the present situation, $\xi=1$ leads to $\left|r_{e e}\right|=0$, which is equivalent to previous results 17 . By using $R_{l, n}^{e e}=0$ and the current conservation low, the conductance results in $G=\left(4 e^{2} / h\right) N_{c}$, where $N_{c}$ is plotted with the dot line in (a). Next we take into account the difference of the effective mass in (b) and that of the Fermi energy in (c), respectively. The results show the sinusoidal MCO and the conductance is at its minimum when $\beta$ is an integer. In (b) and (c), the Andreev reflection is no longer perfect, which can be also explained by the S-matrix in one-dimensional Sm-S junctions, i.e., $\xi \neq 1$. In one-dimensional junctions, the normal reflection can be expected to be zero when $\mu_{S} / \mu_{N}=m_{S} / m_{N}$ and $V_{b h}=0$. The situation is almost the same even in the two-dimensional junction. We show the conductance for $\mu_{S} / \mu_{N}=m_{S} / m_{N}=2.0$ in (a) with the dash line. The MCO disappears and the weak conductance step again can be seen as well as the solid line. In Fig. 11(d), we examine the effects of the potential barrier at the interface, where $V_{b h}=0.5$. The potential barrier is one of the origin of the normal reflection at the interface. We have confirmed for large $V_{b h}$ that the MCO appears even when $\mu_{S} / \mu_{N}=m_{S} / m_{N}$. Thus we conclude that the MCO appears when both the normal and the Andreev reflection occur at the Sm-S interface, (i.e., $\left.|| r_{e e}\right|^{2}-\left.\left|r_{h e}\right|^{2}\right|^{2} \ll 1$ ), irrespective of the origin of the normal reflection. We should note that the S-matrix in the one-dimensional junctions well explains the characteristic feature of the normal and Andreev reflection in the two-dimensional systems although the dimensionality is different in the two systems. The range of the magnetic fields which satisfy the equation

$$
W / 2<L_{c}<W
$$

is denoted by $\leftrightarrow$ in Fig. 1(b)-(d), where $L_{c} \equiv 4 \beta / k_{F}$ is the diameter of the cyclotron orbit. The MCO can be seen when Eq. (蛋) holds, which indicates that the cyclotron motion of a QP plays an important role in the MCO. We have confirmed that the oscillations range shifts to the lower magnetic fields as increasing the wire width, which has been also reported in Ref. 7. In fact, we calculate the amplitude of the wavefunction reflected into the hole branch, $P_{h}(x, y)$ as shown in Fig. 2. Here we focus on the system in Fig. II (d) and fix the magnetic field at $\beta=8.0$ in (a) and 5.5 in (b), respectively. In the dark area, the wavefunction has the large amplitudes. An electronlike $\mathrm{QP}$ is injected from the lower left corner. It is important that an incident wave does not have uniform distribution at the Sm-S interface but has the large amplitudes at several points, as indicated by the arrows. The classical cyclotron orbits are represented by the circles which are drawn to fit the interference pattern for $y<0$ and to pass the reflection points at the interface. It is possible to draw another circles in the figure. We omit them to avoid complexity. Under the magnetic field, it is well known that an electron localizing near the edge of the wire has the larger velocity in the $x$ direction than that localizing around the center of the wire. Thus at $y / W=$ -0.4 , the wave is reflected almost perpendicular to the interface, which corresponds to the fact that the lowest circle is symmetric about $x=0$ as in (a) and (b). The waves at another reflection points have the velocity in the $y$ direction, which allows the asymmetric circle about $x=0$. The figures of Fig. 2 show that the motion of a QP is characterized well by the classical cyclotron orbits near the Sm-S interface when Eq. (4) is satisfied.

Based on the numerical results, we make clear the physical picture of the conductance oscillations within a phenomenological argument. In the range of the magnetic fields in Eq. (4), an incident QP from 2DEG can be scattered twice at the Sm-S interface as shown in Fig. 3. At first an incident electron is either reflected into the electron or the hole branches at $\mathbf{r}_{1}$. After the ballistic motion along the cyclotron orbit $\left(\mathbf{r}_{N}\right)$, the quasiparticle in each branch is reflected again into the electron and hole branches at $\mathbf{r}_{2}$. Thus an incident QP divides into four parts as shown in Fig. 3. In (1) and (3) ( (2) and (4) ), a reflected QP goes across the wire in the electron (hole) branch. The phase of a QP is changed by the magnetic field while traveling along $\mathbf{r}_{N}$. When a QP is in the electron branch, the phase change due to the magnetic field is given by $\phi_{B}=(e / \hbar c) \int_{\mathbf{r}_{1}}^{\mathbf{r}_{2}} d \mathbf{r}_{N} \cdot \mathbf{A}\left(\mathbf{r}_{N}\right)=-\pi \beta$. The phase change of a $\mathrm{QP}$ in the hole branch is given by $-\phi_{B}$. In the following, we separate the reflection process into three steps. We describe the two reflections at the Sm-S interface by using the S-matrix in one-dimensional junctions. The effects of the two-dimensionality and those of the magnetic fields are taken into account through the phase shift by the magnetic field. In this way, we estimate the wavefunction of the four parts as follows,

$$
\begin{aligned}
& \Psi_{1}^{e}=\left|r_{e e}\right| \mathrm{e}^{i \theta_{n}} \cdot \mathrm{e}^{i \phi_{B}} \cdot\left|r_{e e}\right| \mathrm{e}^{i \theta_{n}}, \\
& \Psi_{2}^{e}=\left|r_{e h}\right| \mathrm{e}^{-i \pi / 2} \cdot \mathrm{e}^{-i \phi_{B}} \cdot\left|r_{h e}\right| \mathrm{e}^{-i \pi / 2},
\end{aligned}
$$




$$
\begin{aligned}
& \Psi_{3}^{h}=\left|r_{h e}\right| \mathrm{e}^{-i \pi / 2} \cdot \mathrm{e}^{i \phi_{B}} \cdot\left|r_{e e}\right| \mathrm{e}^{i \theta_{n}}, \\
& \Psi_{4}^{h}=\left|r_{h h}\right| \mathrm{e}^{-i \theta_{n}} \cdot \mathrm{e}^{-i \phi_{B}} \cdot\left|r_{h e}\right| \mathrm{e}^{-i \pi / 2} .
\end{aligned}
$$

The two parts in the electron branch interfere with each other and $\left|\Psi_{1}^{e}+\Psi_{2}^{e}\right|^{2}$ represents the reflection probability as an electron. In the same way, $\left|\Psi_{3}^{h}+\Psi_{4}^{h}\right|^{2}$ is the reflection probability as a hole.

In Fig. 3, we have assumed that the motion of a QP near the interface is characterized by the single cyclotron orbit. The amplitude of the reflected wave in Fig. 2, however, show a number of the circles. When the magnetic field is weak in Fig. 2(a), only the trajectory drawn with the solid circle can contribute to the MCO. A QP can return to the interface after the cyclotron motion along the solid circle. While another circles go across the wire wall before reaching the interface. Thus we can neglect the contribution of these orbits to the MCO. When the magnetic field is relatively strong in Fig. 2(b), most of the reflected wave can contribute to the MCO. The amplitude of the MCO increases with decreasing $\beta$ as shown in Fig. 1. Within the range of the magnetic fields in Eq. (14), the contribution ratio, $p(\beta)$, is set to be unity at $W / 2=L_{c}$ and is zero at $W=L_{c}$. We approximately describe $p(\beta)$ by using a linear function of $\beta$ as $p(\beta)=2\left(1-4 \beta /\left(W k_{F}\right)\right)$. The conductance can be estimated by

$$
\begin{aligned}
G & \simeq g_{0}\left[1-p(\beta)^{2}\left|\Psi_{1}^{e}+\Psi_{2}^{e}\right|^{2}+p(\beta)^{2}\left|\Psi_{3}^{h}+\Psi_{4}^{h}\right|^{2}\right] \\
& \simeq g_{0}\left[1+4\left|r_{e e}\right|^{2}\left|r_{h e}\right|^{2} p(\beta)^{2} \cos \left(2 \pi \beta-2 \theta_{n}\right)\right] .
\end{aligned}
$$

where $g_{0}=\left(2 e^{2} / h\right) N_{c}$ and we use the relation $\left(\left|r_{e e}\right|^{2}-\left|r_{h e}\right|^{2}\right)^{2} \ll 1$. In real space, the cyclotron orbits in Fig. 3 do not encircle the magnetic flux. However, the phase shift by the magnetic field in $\Psi_{1}^{e}$ and $\Psi_{3}^{h}$ have the opposite sign to that in $\Psi_{2}^{e}$ and $\Psi_{4}^{h}$. Thus the magnetic field causes the interference effect. In Fig. 3, we only consider the symmetric orbits about $x=0$. The numerical results show a number of the asymmetric circles. In Fig. 2(b), for instance, an incident QP is reflected into the two branches at $y / W=-0.28$. When the QP travels along $O_{1}$ in the hole branch, the corresponding part in the electron branch travels along $\mathrm{O}_{2}$. We note that the $O_{1}$ and $O_{2}$ are symmetric with each other about $x=0$. This is because, only the velocity component perpendicular to the interface changes sign in the normal reflection, however, all velpcity components change sign in the Andreev reflection 3 . It can be easily confirmed that the difference in the phase shift between $O_{1}$ in the hole and $\mathrm{O}_{2}$ in the electron branches is equivalent to $2 \phi_{B}$. Thus the asymmetric orbits contribute to MCO as well as the symmetric orbits and the phase shift remains constant independent of the incident angle of a QP to the Sm-S interface. A possibility of the conductance oscillations was briefly mentioned in Ref. 7. A part of the argument, however, was not correct. In the absence of the potential barrier, $\theta_{n}$ becomes zero and the conductance is at its maximum when $\beta$ is an integer as shown in Eq. (10). The numerical results, however, show that the conductance takes its minima at these points. The sign of the oscillating part is a disagreement between the simple analysis and the numerical results. Since we do not explicitly consider the two-dimensionality, the disagreement may stem from the wavefunction in the $y$ direction. At present, we can not give a satisfactory explanation of the disagreement. In Fig. 1(b)-(d), we compare Eq. (10) (dash line) with the numerical results. The results show an excellent agreement with each other. Here we have to confess that the sign of the second term in Eq. (10) has been changed from + to - in Fig. 1.

We conclude that the interplay between the classical cyclotron motion of a quasiparticle and the phase shift by the magnetic field is the origin of the magnetoconductance oscillations. The conductance oscillations can be seen even in the simply-connected Sm-S junctions because the charge degree of freedom of an electron restricts an electron wave into the classical cyclotron orbit under the magnetic field and the superconductor opens the hole branch in the 2DEG. Finally we briefly discuss the possibility to observe the MCO in experiments. When the width of the wire is $W=5 \times 10^{-6} \mathrm{~m}$ as it is in Ref. 5 , the $\mathrm{MCO}$ can be seen around $B \sim 0.08-0.16 \mathrm{~T}$. We have assumed the perfect screening of the magnetic field at S. In experiments, the screened magnetic field is not necessary to be zero. In this case, the phase fluctuations in the pair potential are caused by the magnetic field. However, the phase fluctuations within the length scale $L_{c}$ is not so large because the coherence length of $\mathrm{S}$ is larger than $L_{c}$ when $\mathrm{S}$ is type $\mathrm{I}$. In the absence of the magnetic shielding at $\mathrm{S}$, we can show that the MCO is washed out or can be seen as the noise-like fluctuations when $\mathrm{S}$ is type II. The details will be given elsewhere.

The author is indebted to N. Tokuda, H. Akera, T. Kato and Y. Takane for useful discussion.

${ }^{1}$ R. A. Webb, S. Washburn, C. P.Umbach, abd R. B. Laibowitz, Phys. Rev. Lett. 54, 2696 (1985).

${ }^{2}$ Y. Aharonov and D. Bohm, Phys. Rev. 115, 485 (1959).

${ }^{3}$ P. A. M. Benistant, H. van Kempen, and P. Wyder, Phys. Rev. Lett. 51, 817 (1983).

${ }^{4} \mathrm{H}$. van Houten, B. J. van Wees, J. E. Mooij, C. W. J. Beenakker, J. G. Williamson and C. T. Foxon, Europhys. Lett. 5, 721 (1988).

${ }^{5}$ H. Takayanagi and T. Akazaki, Physica B 249-251, 462 (1998).

${ }^{6}$ A. F. Andreev, Zh. Eksp. Theor. Fiz, 46, 1823 (1964) [Sov. Phys. JETP 19, 1228 (1964)].

${ }^{7}$ Y. Takagaki, Phys. Rev. B 57, 4009 (1998).

${ }^{8}$ B. J. van Wees, P. de Vries, P. Magnee, and T. M. Klapwijk, Phys. Rev. Lett. 69, 510 (1992).

${ }^{9}$ C. W. J. Beenakker, J. A. Melsen, and P. W. Brouwer, Phys. Rev. B 53, 14530 (1995). 
${ }^{10}$ Yu. Nazarov and T. H. Stoof, Phys. Rev. Lett. 76, 823 (1996).

${ }^{11}$ A. F. Morpurgo, S. Holl, B. J. van Wees, T. M. Klapwijk, and G. Borghs, Phys. Rev. Lett. 78, 2636 (1997).

${ }^{12}$ P. G. de Gennes Superconductivity of Metals and alloys, (Benjamin, New York, 1966).

${ }^{13}$ U. Schüssler and R. Kümmel, Phys. Rev. B47, 2754 (1993).

${ }^{14}$ Here we assume type I superconductors.

${ }^{15}$ H. Tamura and T. Ando, Phys. Rev. B44, 1792 (1991).

${ }^{16}$ Y. Takane and H. Ebisawa, J.Phys. Soc. Jpn. 61, 1685 (1992).

${ }^{17}$ G. E. Blonder, M. Tinkham and T. M. Klapwijk, Phys. Rev. B 25, 4515 (1982).

FIG. 1. The conductance is numerically calculated as a function of the inverse of the magnetic field. The number of the propagating channels in 2DEG, $N_{c}$, is plotted by the dot line in (a). In (a), the normal reflection does not occur at the Sm-S interface. While the normal reflection occurs at the interface and the conductance oscillations appear in (b), (c) and (d). In (b)-(d), we compare the analytic results (dash line) with the numerical results.

FIG. 2. The amplitudes of the wavefunction reflected into the hole branch are shown for the system discussed in Fig. 1(d). We fix the magnetic field at $\mu_{N} / \hbar \omega_{c}=8.0(\mathrm{a})$ and 5.5 (b), respectively. The classical cyclotron orbits are represented by the circles.

FIG. 3. Schematic picture of the reflection process from the Sm-S interface. The solid and broken lines denote a quasiparticle in the electron and hole branches, respectively. 


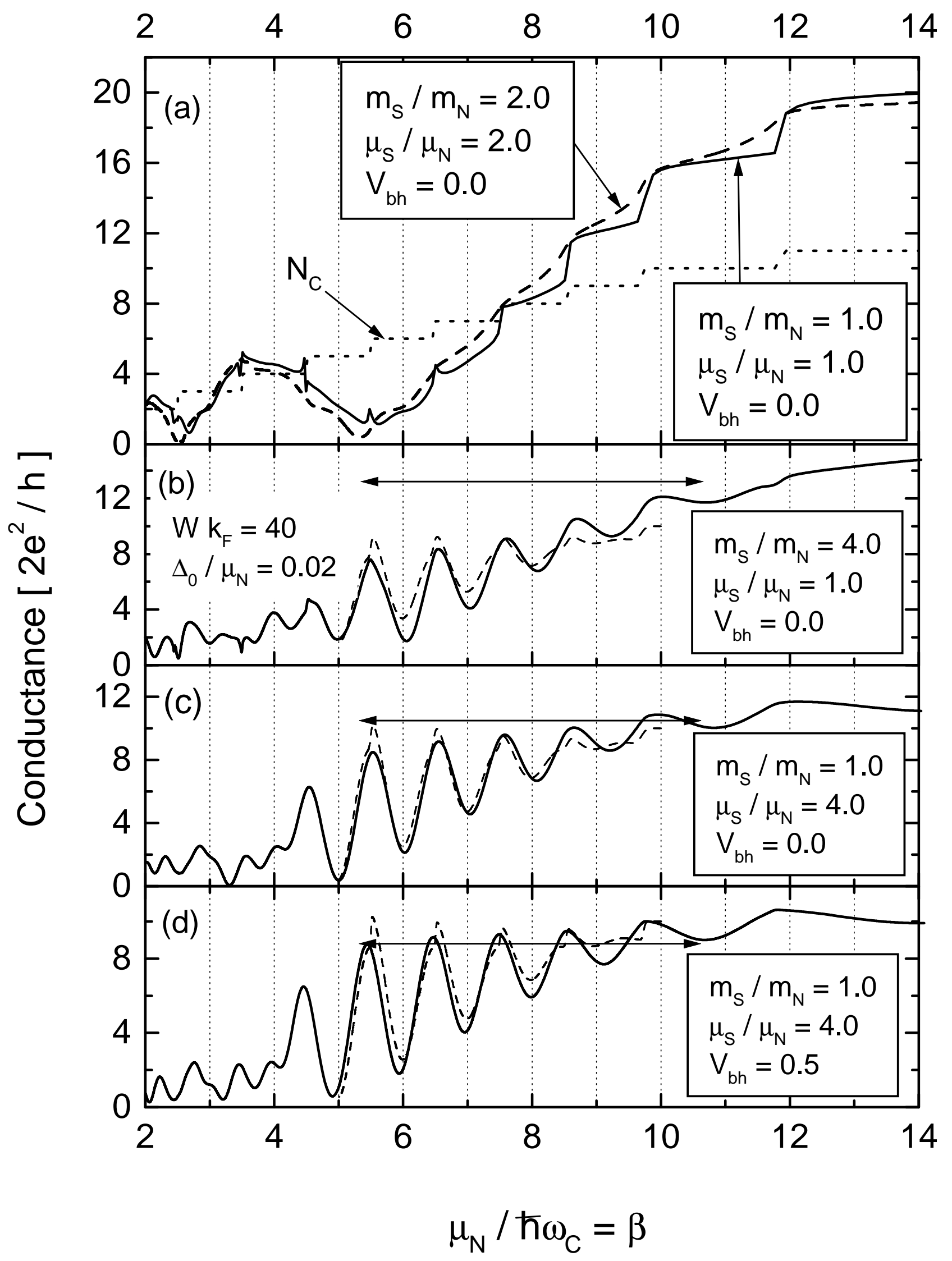

Fig. $1(a)(b)(c)(d)$, Asano 


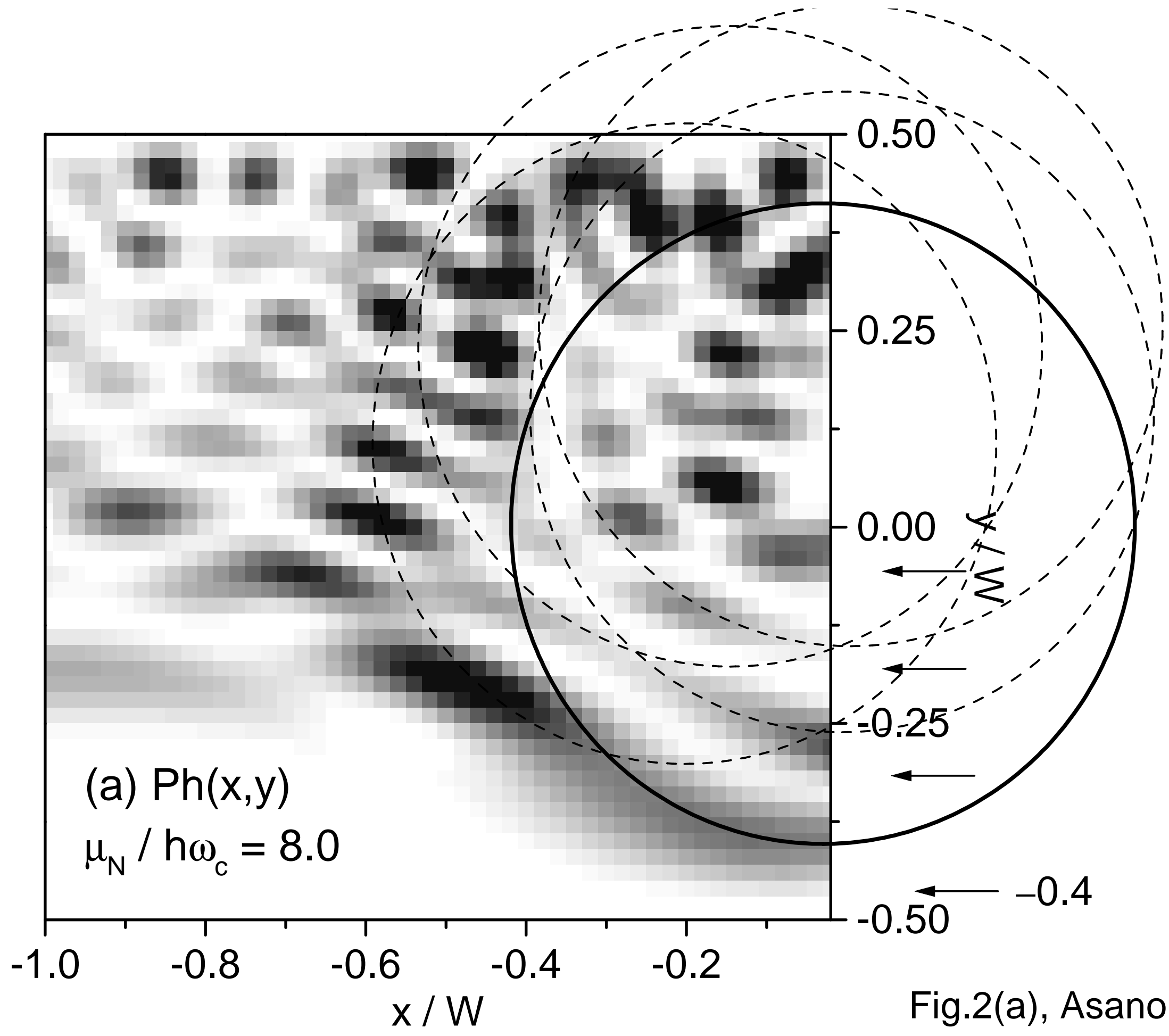




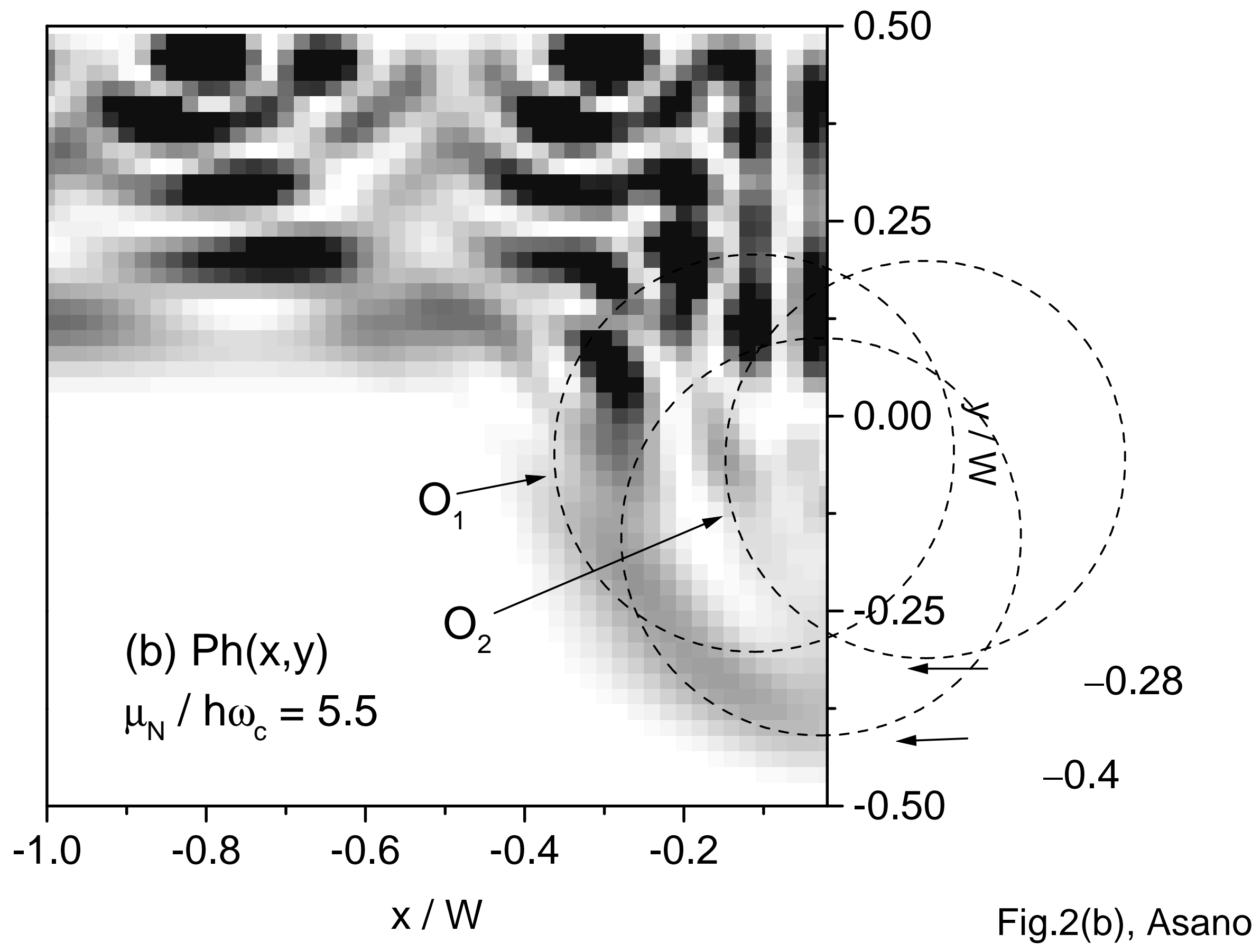


Semiconductor

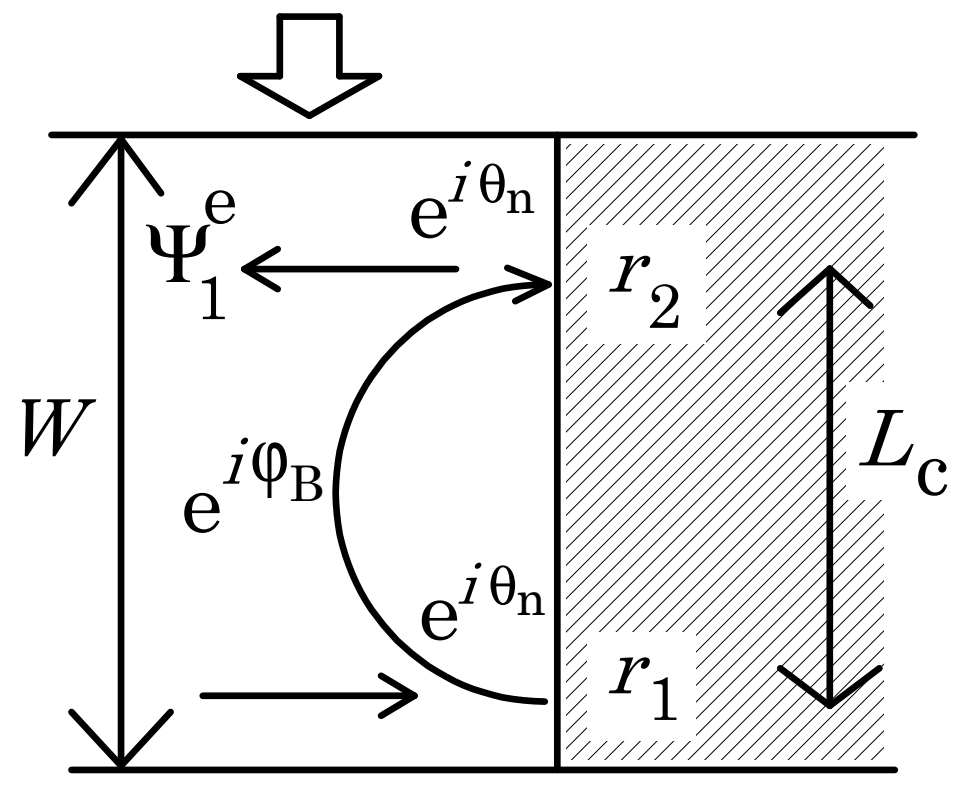

(1)

$\rightarrow$ electron

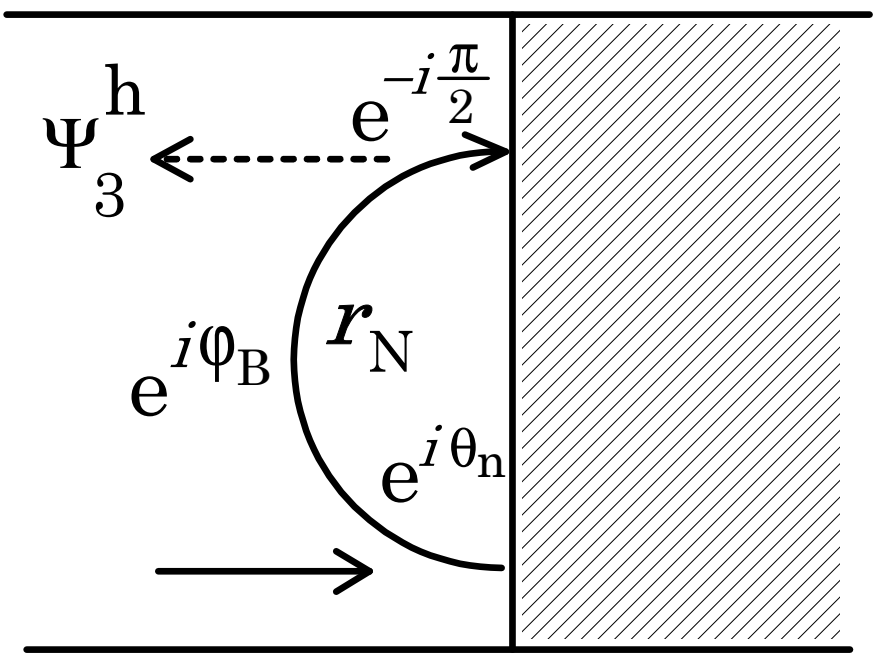

(3)
Superconductor
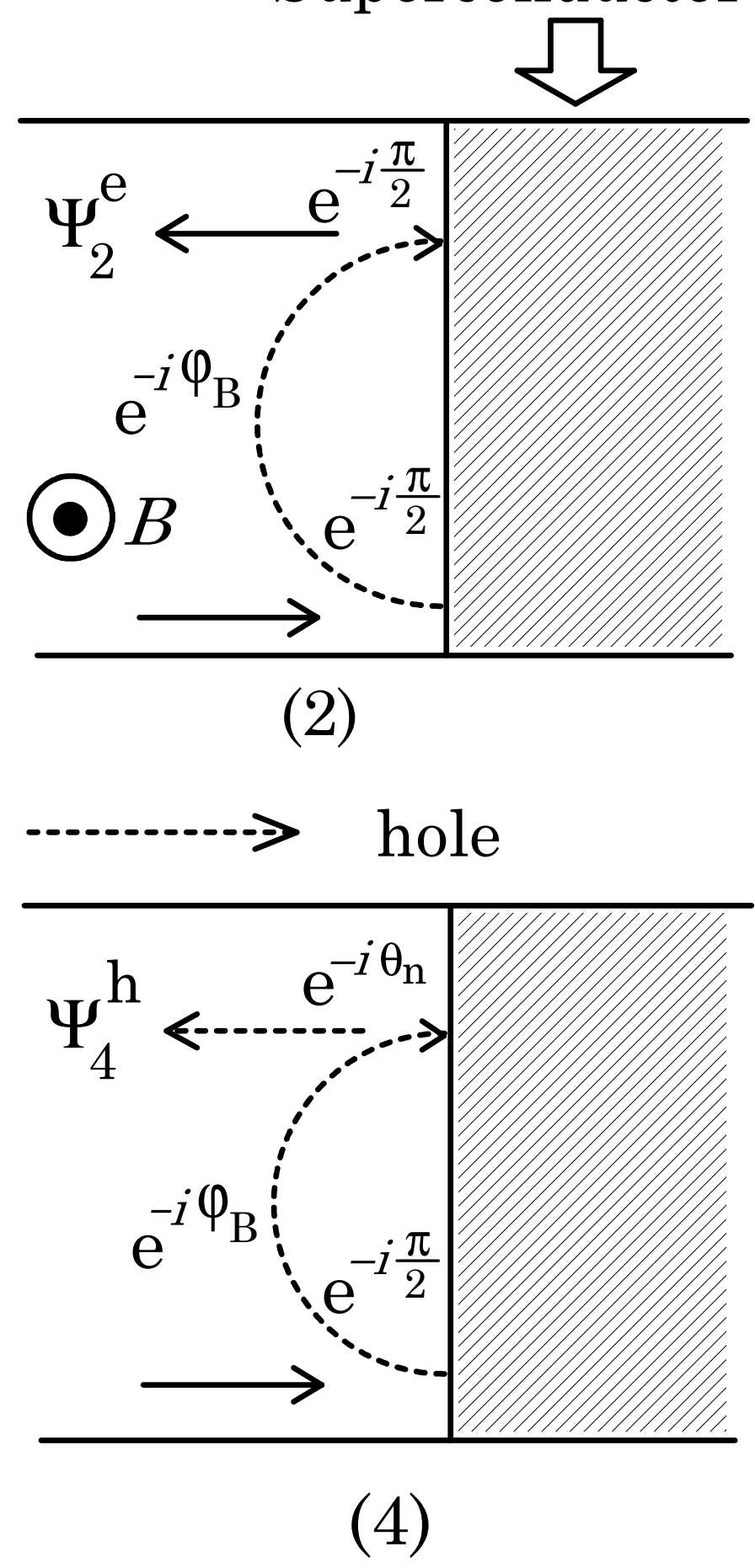

Fig. 3, Asano 